\title{
MEASUREMENT OF THE FEMORAL NECK ANGLE IN MEDIUM AND LARGE DOG BREEDS USING COMPUTED TOMOGRAPHY
}

\author{
Ahmad Al AIYAN $^{1 *}$, Kenneth RICHARDSON ${ }^{2}$, George MANCHI $^{3}$, Johanna PLENDL ${ }^{4}$ \\ and Leo BRUNNBERG ${ }^{3}$ \\ ${ }^{1}$ Department of Veterinary Medicine, College of Food and Agriculture, United Arab \\ Emirates University, P.O. Box 15551, Al Ain, Abu Dhabi, United Arab Emirates; \\ ${ }^{2}$ College of Veterinary Medicine, School of Veterinary and Life Sciences, Murdoch \\ University, Perth, Australia; ${ }^{3}$ Department of Veterinary Medicine, Small Animal Clinic, \\ Free University of Berlin, Germany; ${ }^{4}$ Department of Veterinary Medicine, \\ Institute of Veterinary Anatomy, Free University of Berlin, Germany
}

(Received 15 November 2018; accepted 15 February 2019)

The aim of this study was to get precise normal values of the femoral neck angle (FNA) in support of developing an optimally functioning total hip prosthesis for medium and large dog breeds. Accordingly, two- and three-dimensional computed tomographic images of the anatomical structures of the proximal femora of 58, hip-dysplasia-free, mature dogs of medium and large breeds were studied. Based on the length of their femora the dogs were allocated to Group I (from 145 to $195 \mathrm{~mm}$ ) and Group II (from 196 to $240 \mathrm{~mm}$ ). The FNA was measured on each femur using multi-slice spiral computed tomography (CT). The two- and three-dimensional image data were processed as multi-planar and threedimensional reconstructions using Advantage Workstation software. The CT measurements revealed that Group I had an average femoral neck angle of $147.59^{\circ}$ (min. $144.05^{\circ}$, max. $153.35^{\circ}$ ), while in Group II the average FNA was $147.46^{\circ}$ (min. $141^{\circ}$, max. $154.35^{\circ}$ ). There was no significant correlation between the length of the femur and the FNA in either group. The optimal FNA for a total hip prosthesis is $147.5^{\circ}$ for medium and large dog breeds.

Key words: Canine, total hip replacement, femoral morphology, femoral neck angle, total hip prosthesis

In the clinical assessment of hip joint health, the femoral neck angle (FNA) is a highly significant and frequently used measure (Adams et al., 2017). Here the FNA is defined as the angle formed by the intersection of the longitudinal axis of the femoral shaft and that of the femoral neck (Hauptman et al., 1979) (Fig. 1). In adult medium to large dog breeds with normal hip joint morphology the FNA has been reported to be within the range of $129.11^{\circ}$ to $153.8^{\circ}$ degree

*Corresponding author; E-mail: a.alaiyan@uaeu.ac.ae; Phone: 00971-3713-4556 
(Hauptman et al., 1979; Hauptman et al., 1985; Sumner et al., 1990; Martins et al., 2012; Lusetti et al., 2017). However, there are many relatively common congenital and developmental conditions where the FNA deviates significantly from the normal such as coxa valga where the FNA is above the normal, and coxa vara where the FNA is lower than normal (Adams et al., 2017).

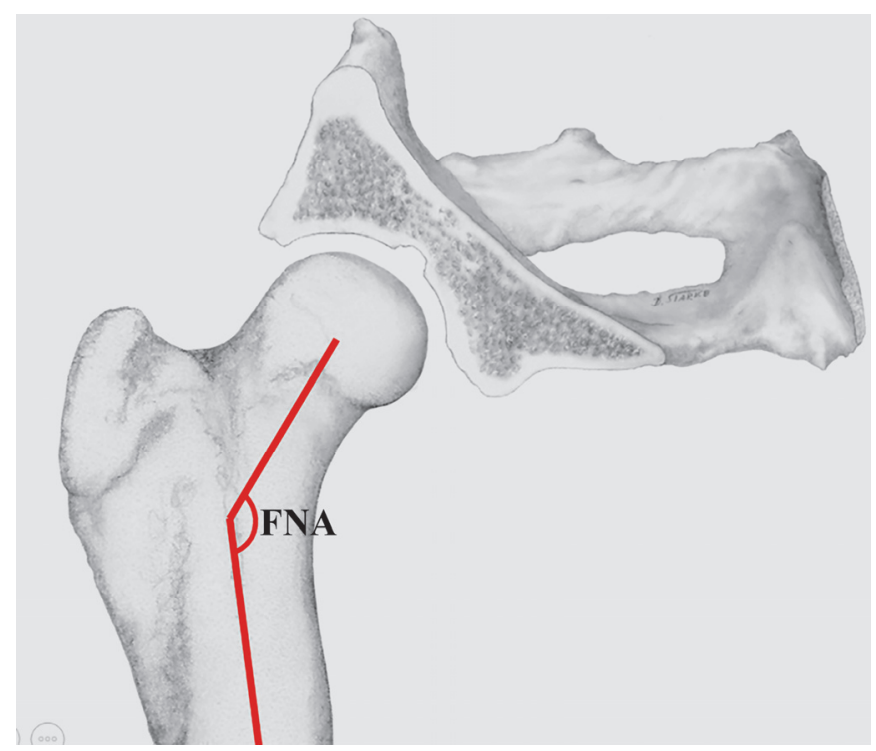

Fig. 1. An illustration of the normal position of the femoral head within the acetabulum in a dog with a normal femoral neck angle (FNA)

Surgical correction of serious hip problems frequently requires total hip replacement (Dudley et al., 2006; Bausman and Wendelburg, 2013). For these, a detailed knowledge of the morphology of the dog's proximal femur is required for the development of an ideal hip joint prosthesis (Noble et al., 1988; Sumner et al., 1990; Palierne et al., 2006). The accurate colocation of the femoral head with the acetabulum is critical in healthy dogs; hence, the correct angulation of the femoral head and neck is an important factor in the development of total hip prostheses (Sumner et al., 1990; Palierne et al., 2006). Accurate measurements of the FNA are important for understanding the geometric configuration of the bony joint structures and the biomechanics of the hip joint, so that the design of total hip prosthesis can be optimised (Hauptman et al., 1985; Sumner et al., 1990; Palierne et al., 2006).

Historically the FNA has been measured using a variety of techniques including routine radiography (Montavon et al., 1985; Sumner et al., 1990; Sarierler, 2004; Palierne et al., 2006), and more recently two-dimensional (2D) (Ocal et al., 2004; Bäcker, 2010; Lusetti et al., 2017) and three-dimensional (3D) recon- 
structed computed tomography (CT) (Hartel et al., 2016; Savio et al., 2016). Measurement of the FNA using routine classical radiographic methods can be technically challenging and time consuming as well as being subject to operator experience and biases (Dudley et al., 2006; Jackson and Wendelburg, 2012). Establishing the femoral axis is the starting point for the cascade of measures to ultimately determine the FNA (Tomlinson et al., 2007). However, these measurement points are poorly defined in X-ray images due to superimposition-related errors when compared to those of CT images (Bäcker, 2010).

The greater clarity of structural images using $\mathrm{CT}$ allows a precise determination of the medullary axis of the femur; this in turn allows more accurate determination of the subset of femoral head and neck measurements that are needed for the development of total hip prostheses (Bäcker, 2010; Savio et al., 2016). We used CT to get precise normal values of the FNA in medium and large dog breeds in order to develop an optimally functioning total hip prosthesis for these dog breeds.

\section{Materials and methods}

Computed tomographic studies were conducted on the proximal femora of 58 mature dogs, all free of hip dysplasia, that had died or were euthanised for medical reasons at the Small Animal Clinic, Free University of Berlin in accordance with the research ethics code of the institution and with the written permission of the animal owner. Each dog was weighed to an accuracy of $0.01 \mathrm{~kg}$ on a digital scale (Animal Platform Scale, Hitsan). Dogs were assigned into two groups based on the length of their femur (Palierne et al., 2008). Group I had 25 dogs, whose femoral length was between 145 and $195 \mathrm{~mm}$, while Group II included 33 dogs with a femoral length between 196 and $240 \mathrm{~mm}$.

The German Shepherd was the most frequent breed with an almost balanced sex ratio, followed by Labradors and Golden Retrievers. Other breeds, such as Staffordshire Terriers, Boxers, Rottweilers, Bullmastiffs and Weimaraners, were less frequent in this study. The dogs used in this study included 17 castrated females, 4 intact females, 2 castrated males and 35 intact males.

To ensure that the animals used in this study were normal and free of any orthopaedic disease, we performed the Ortolani and Barlow test on the live dogs before their euthanasia, while radiographic and computer tomographic evaluations of the hip joint were conducted on all studied dogs after their euthanasia. None of the dogs studied had a clinical history of hindlimb lameness as reported by the owners, moreover all hip joints that had radiographic signs of hip joint dysplasia on CT examination were excluded. The FNA was measured using twoand three-dimensional CT at the Small Animal Clinic, Free University of Berlin. The CT used multi-slice spiral computed tomography 'Lightspeed' QXi (General Electric Healthcare, GE). The slice thickness selected was $(0.3 \mathrm{~mm})$, the kilovoltage setting was $120 \mathrm{kV}$ and the milliampere-seconds (mAs) setting was $130 \mathrm{mAs}$. 


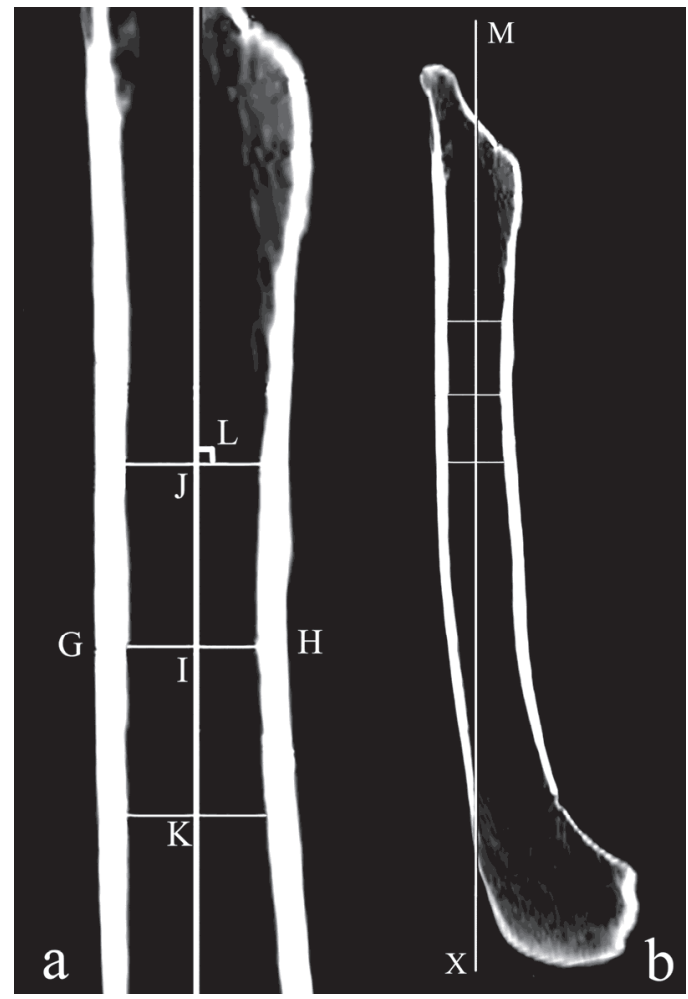

Fig. 2. Sagittal plane view of the femur where (a) is a high resolution of the proximal region and (b) is an image of the complete femur. Here GH is the intracortical width at the narrowest point of the femoral shaft and $\mathrm{I}$ is its centre point. $\mathrm{J}$ and $\mathrm{K}$ are central points $2 \mathrm{~cm}$ proximal and $2 \mathrm{~cm}$ distal to I, respectively. MX is the medullary axis. $\mathrm{L}$ is the right angle between the medullary axis and the width $(\mathrm{GH})$

The dogs were positioned on their back on the CT scanner's table and, to avoid rotation of the femoral bones, the hindlimbs were fixed with adhesive strips (Tesa AG Hamburg) at the level of the tarsal joint.

The images were analysed using a workstation (Advantage Workstation 4.2; GE Healthcare). The data record was processed as multi-planar and threedimensional reconstructions using the same Advantage Workstation software.

The sequence of femoral measurements to determine the axis of the femoral shaft, length of the femur, centre of the femoral head, axis of the femoral neck and the femoral neck angle were always performed in the same order as some measurements were reliant on values of earlier measurements. To ensure precision, all measurements were performed twice at a time interval of one day, and then the mean values determined.

The data were analysed statistically by using Statistical Packages for Social Sciences software (SPSS Inc. Version 20, Chicago IL, USA). 


\section{Medullary axis of the femoral shaft}

To ensure accurate comparability in femoral measurements sagittal plane views were all made with the caudal aspects of both femoral condyles at the same level and the femoral axis positioned vertically to avoid any cranial or caudal inclination of the femur (Fig. 2). From here the femora were rotated $90^{\circ}$ cranially to ensure an accurate frontal plane view and to avoid any external or internal rotation of the femur.

In the frontal plane view, the centre $(C)$ of the intracortical width $(A B)$ was established at the narrowest point of the femoral shaft. Using the same methodology an additional central point (D) was determined $2 \mathrm{~cm}$ proximal to $\mathrm{C}$ and a third central point (E) was determined $2 \mathrm{~cm}$ distal to $\mathrm{C}$ (Fig. 3).

The axis of the femoral shaft was defined as the line connecting the points $\mathrm{C}, \mathrm{D}$ and $\mathrm{E}$ that forms a right angle to width $\mathrm{AB}$ (Fig. 3).

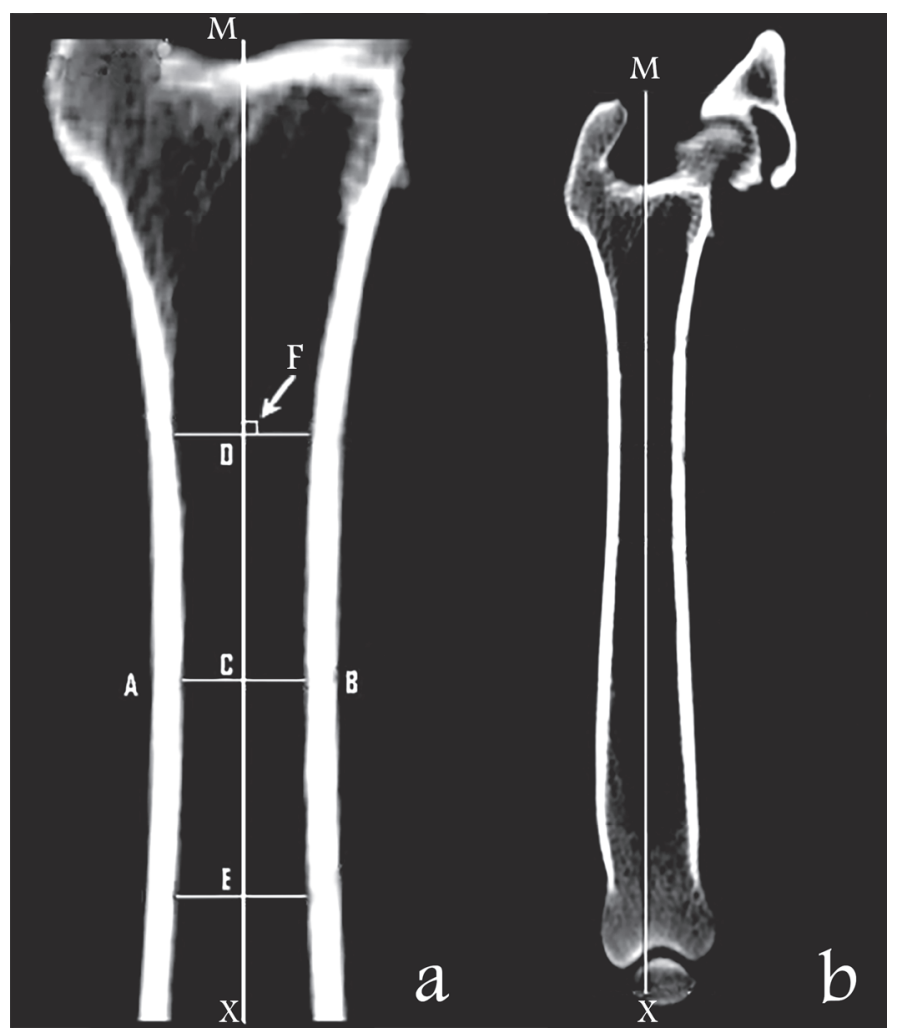

Fig. 3. Frontal plane view of the femur where (a) is a high resolution of the proximal region and (b) is an image of the complete femur. Here $\mathrm{AB}$ is the intracortical width at the narrowest point of the femoral shaft and $\mathrm{C}$ is its centre point. $\mathrm{D}$ and $\mathrm{E}$ are central points $2 \mathrm{~cm}$ proximal and $2 \mathrm{~cm}$ distal to $\mathrm{C}$, respectively. MX is the medullary axis. $\mathrm{F}$ is the right angle between the medullary axis and the width $(\mathrm{AB})$ 


\section{Length of the femur}

Using a three-dimensional rendering of the femur in frontal plane view, the length of the femur was determined to be the line immediately parallel to the femoral axis that connects the orthogonal lines at the most proximal point of the femoral head and at the most distal end of the femoral condyles (Fig. 4). The length of the femur was used to allocate the dogs into Group I (from 145 to $195 \mathrm{~mm}$ ) and Group II (from 196 to $240 \mathrm{~mm}$ ).

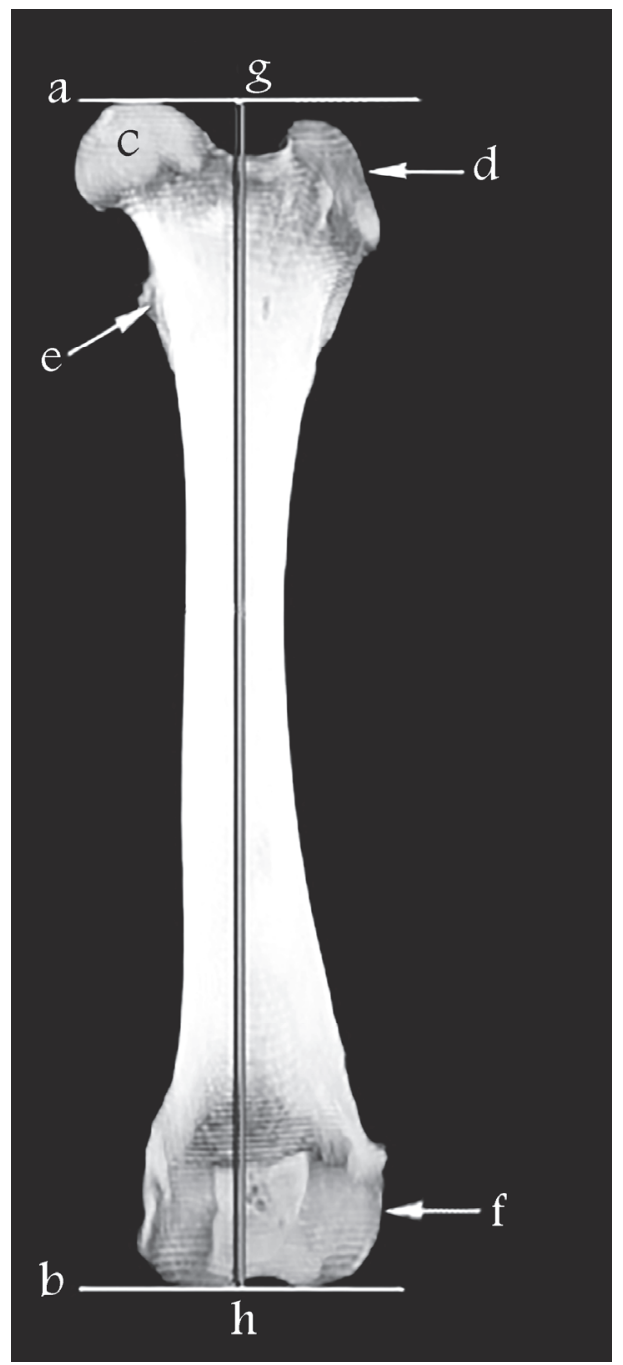

Fig. 4. Frontal plane view of the femur: (a) proximal orthogonal, (b) distal orthogonal, (c) femoral head, (d) great trochanter, (e) lesser trochanter, (f) lateral condyle, (gh) length of the femur connecting the proximal orthogonal at $(\mathrm{g})$ to the distal orthogonal at (h) 


\section{Centre of the femoral head}

Using a 3D rendering of the femur in frontal plane view, the centre of the femoral head was identified by using annotation software to generate concentric circles of best fit and superimpose these onto the femoral head. The circle of best fit was identified and its centre marked the centre of the femoral head (Fig. 5).

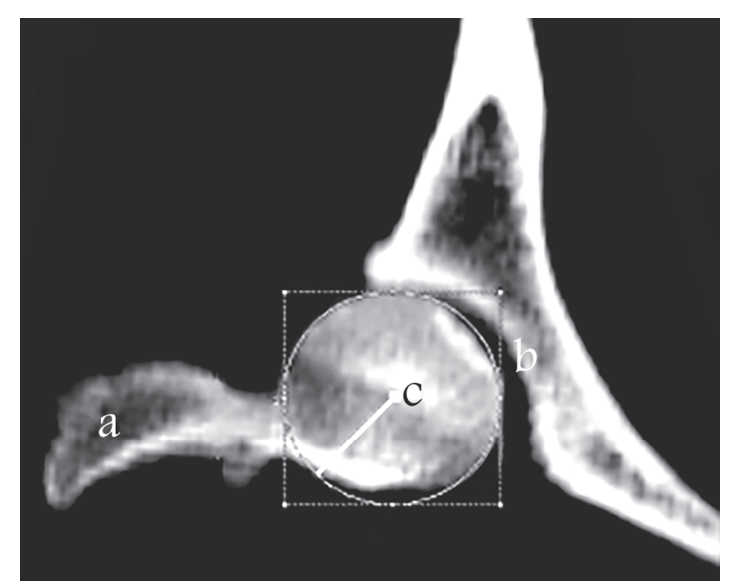

Fig. 5. Frontal plane view of the proximal femur: (a) great trochanter, (b) acetabular cavity, (c) centre of the femoral head

\section{Axis of the femoral neck}

The axis of the femoral neck was defined as the line passing from the centre of the femoral head to form a right angle at the midpoint of the base of the femoral neck. This is on the line between the deepest point of the trochanteric fossa and the medial cortex of the femur at the level of the lesser trochanter (Fig. 6). Here the centre of the femoral head and the midpoint of the base of the femoral neck are located in two different frontal plane slices due to the cranial femoral head and neck antetorsion to the femoral shaft. This explains the difference in shape of the femoral head in Figs 5 and 6.

\section{Femoral neck angle}

The FNA is the angle between the axis of the femoral neck and the medullary axis of the femoral shaft (Fig. 7).

\section{Results}

In this study, 116 femora in 58, hip-dysplasia-free, mature dogs of medium and large breeds were measured. 


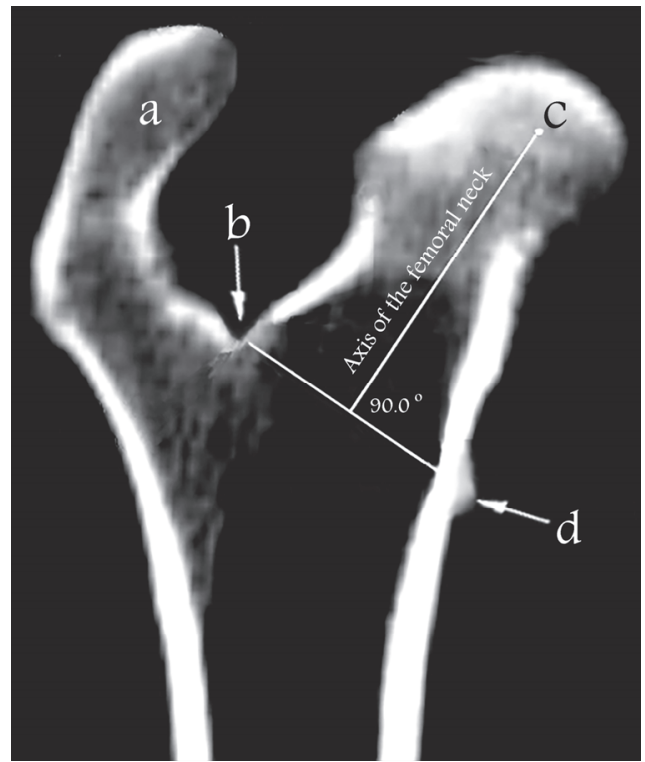

Fig. 6. Frontal plane view of the femur: (a) great trochanter, (b) deepest point of the trochanteric fossa, (c) centre of the femoral head, (d) lesser trochanter. The axis of the femoral neck is the line drawn from the centre of the femoral head to be orthogonal to the line joining (b) and (d)

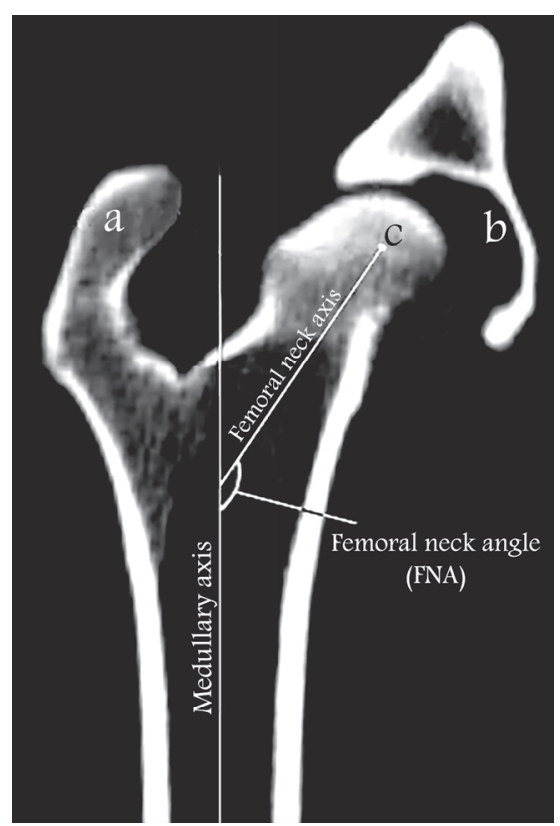

Fig. 7. Frontal plane view of the femur: (a) great trochanter, (b) acetabular cavity, (c) centre of the femoral head. The femoral neck angle (FNA) lies between the femoral neck axis and the medullary axis of the femur 
In Group I, the average age of the dogs was 7.6 years with a minimum of 2 years and a maximum of 16 years, while the average body weight was $27.8 \mathrm{~kg}$ with a minimum of $17 \mathrm{~kg}$ and a maximum of $45 \mathrm{~kg}$. The average age of the dogs in Group II was 8.4 years with a minimum of 1.5 years and a maximum of 16 years, while the average body weight was $42.3 \mathrm{~kg}$ with a minimum of $22 \mathrm{~kg}$ and a maximum of $60 \mathrm{~kg}$.

\section{Femoral neck angle (FNA)}

In the present study, the mean FNA values were $147.59^{\circ}(\mathrm{SD} \pm 2.24$, range $\left.144.05^{\circ}-153.35^{\circ}\right)$ for Group I and $147.46^{\circ}\left(\mathrm{SD} \pm 3.05\right.$, range $141^{\circ}-$ $154.35^{\circ}$ ) for Group II. The comparison of the measured values of the FNA in box plot (Fig. 8) revealed no significant difference between Groups I and II, and no correlation between the length of the femur and the femoral neck angle.

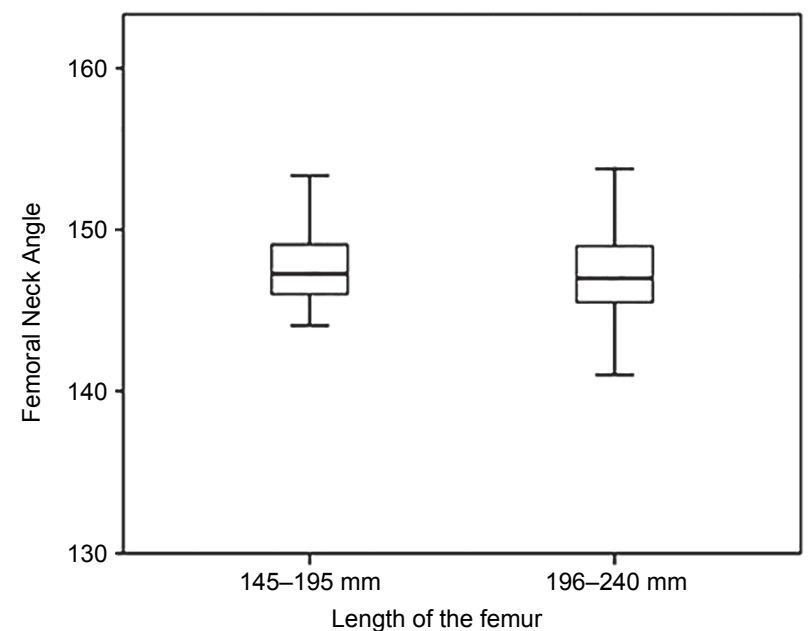

Fig. 8. Femoral neck angle (FNA) and length of the femur in Groups I and II. Results are presented as a boxplot with medians and data ranges

\section{Discussion}

Since the first hip prosthetic implant in a dog with hip dysplasia in 1974 (Olmstead et al., 1983), total hip arthroplasty in veterinary medicine has been evolving continuously (Guerrero and Montavon, 2009). The design and modularisation of hip prostheses as well as the techniques of implantation have been the subject of many studies (Sumner et al., 1990; Bloebaum et al., 1993; Palierne et al., 2006; Bausman and Wendelburg, 2013). Complications following hip joint replacement include aseptic loosening, implant subsidence, infection, and im- 
plant-associated fracture; however, hip luxation was and continues to be the most frequently reported complication for both cemented and uncemented hip prostheses (Bergh et al., 2006; Nelson et al., 2007; Guerrero and Montavon, 2009; Bausman and Wendelburg, 2013). Consequently, detailed knowledge of the normal values of the geometric parameters of the femur support the recognition, understanding and treatment of pathologic conditions of the hip joint. This in turn allows the development of optimally functioning hip joint prostheses that closely mimic the hip joint architecture, thus playing an important role in achieving favourable functional outcomes (Tonnis and Heinecke, 1999; Hartel et al., 2016). This knowledge improves long-term success rates and reduces postoperative complication rates such as cortical bone loss, fractures and prosthesis luxation by ensuring optimal positioning of the hip prosthesis (Guerrero and Montavon, 2009). Like Palierne et al. (2008) who studied a large number of dogs of quite different body sizes we did not include the body mass of the dogs as a morphological parameter due to the variability in their history and nutritional status. In their study of femoral characters such as the size of the femoral head, FNA, and the length of the femoral neck they found that the total length of the femur was the most reliable parameter to divide the dog population into similar functional size groups (Palierne et al., 2008).

The FNA is described as one of the most important parameters of the proximal end of the femur in the development of hip dysplasia (Prieur, 1980; Adams et al., 2017). Some studies have reported that there is no difference in the FNA of dysplastic and non-dysplastic dogs (Hauptman et al., 1985; Sarierler, 2004). Contrary to this, however, other studies report that there is a greater FNA in dogs with hip dysplasia (Prieur, 1980; Montavon et al., 1985; Bloebaum et al., 1993). Most studies measured the FNA for medium and large dog breeds using standard radiographic methods and reported the FNA as follows: Sterchi (1980) $147^{\circ}$, Schawalder and Sterchi (1981) $142^{\circ}$, Hauptman et al. (1985) $143^{\circ}$, Montavon et al. (1985) $144.7^{\circ}$, Sumner et al. (1990) $147.4^{\circ}$. Palierne et al. (2006), who included small, medium and large dog breeds in their study, reported that the average FNA was $140.9^{\circ}$. This lower measure is most probably due to the inclusion of small dogs that have a smaller FNA. This was confirmed by Bäcker (2010) who measured the FNA of small dog breeds using CT and reported an FNA with a mean value of $138.2^{\circ}$.

The Prosthesis Inclination Angle (PIA) is the prosthetic equivalent of the FNA in live dogs and is determined by each implant manufacturer's design. For BioMedtrix $\left(\mathrm{CFX}^{\mathrm{TM}}\right.$ cemented fixation and $\mathrm{BFX}^{\mathrm{TM}}$ biologic fixation systems) it is $135^{\circ}$ and for the Kyon uncemented system it is $145^{\circ}$ (Bausman and Wendelburg, 2013). This angle should match the normal values of the FNA (Bausman and Wendelburg, 2013).

Accurate determination of the femoral neck axis is crucial to the measurement and determination of the FNA (Tomlinson et al., 2007). Any rotation of 
the femur either internally or externally from the true frontal plane, and any error in the sagittal plane position of the femur alters the measurement of the inclination angle in dogs (Beck et al., 1992; Miles, 2016). Elevation of the femur from being horizontal to the X-ray beam also affects the measurement of the anatomical lateral distal femoral angle (Miles et al., 2015). In this study strict adherence to the accepted methodologies nullified any possible sagittal inclination, external or internal rotation of the femur and antetorsion of the femoral neck.

In the present study the mean value of the FNA was $147.59^{\circ}$ in dogs with a femur length between 145 and $195 \mathrm{~mm}$ (Group I) and it was $147.46^{\circ}$ in dogs with a femur length between 196 and $240 \mathrm{~mm}$ (Group II). These results are comparable to those of Sterchi (1980) with an FNA of $147^{\circ}$, Montavon et al. (1985) at $144.7^{\circ}$ and Sumner et al. (1990) at $147.4^{\circ}$. The FNA measured in this study shows no significant difference between Group I and Group II. Likewise, there was no correlation between the length of the femur and the FNA (Fig. 8). According to this study, any new hip joint prosthesis should have an FNA of $147.5^{\circ}$ for both medium and large dog breeds. We emphasise the importance of the FNA, which is critical in the development of properly designed, highly functional total hip prostheses. This will, in turn, improve the long-term success rate and reduce the postoperative complications associated with hip joint replacement.

\section{References}

Adams, R. W., Gilleland, B., Monibi, F. and Franklin, S. P. (2017): The effect of valgus and varus femoral osteotomies on measures of anteversion in the dog. Vet. Comp. Orthop. Traumatol. 30, 184-190.

Bausman, J. A. and Wendelburg, K. L. (2013): Femoral prosthesis version angle calculation from a sagittal plane radiographic projection of the femur. Vet. Surg. 42, 398-405.

Bäcker, C. (2010): The geometric configuration of the hip joint of small breed dogs [in German], abbreviated. PhD Thesis, Freie Universität Berlin.

Beck, K. A., Erb, H. N. and Tapley, K. (1992): Effect of sagittal plane positioning errors on measurement of the angle of inclination in dogs. Vet Surg. 21, 332-336.

Bergh, M. S., Gilley, R. S., Shofer, F. S. and Kapatkin, A. S. (2006): Complications and radiographic findings following cemented total hip replacement: a retrospective evaluation of 97 dogs. Vet. Comp. Orthop. Traumatol. 19, 172-179.

Bloebaum, R. D., Ota, D. T., Skedros, J. G. and Mantas, J. P. (1993): Comparison of human and canine external femoral morphologies in the context of total hip replacement. J. Biomed. Mater. Res. 27, 1149-1159.

Dudley, R. M., Kowaleski, M. P., Drost, W. T. and Dyce, J. (2006): Radiographic and computed tomographic determination of femoral varus and torsion in the dog. Vet. Radiol. Ultrasound. $47,546-552$.

Guerrero, T. G. and Montavon, P. M. (2009): Zurich cementless total hip replacement: retrospective evaluation of 2 nd generation implants in 60 dogs. Vet. Surg. 38, 70-80.

Hartel, M. J., Petersik, A., Schmidt, A., Kendoff, D., Nüchtern, J., Rueger, J. M., Lehmann, W. and Grossterlinden, L. G. (2016): Determination of femoral neck angle and torsion angle utilizing a novel three-dimensional modeling and analytical technology based on CT Datasets. PLoS One 11, e0149480. 
Hauptman, J., Cardinet, G. H., Morgan, J. P., Guffy, M. M. and Wallace, L. J. (1985): Angles of inclination and anteversion in hip dysplasia in the dog. Am. J. Vet. Res. 46, 2033-2036.

Hauptman, J., Prieur, W. D., Butler, H. C. and Guffy, M. M. (1979): The angle of inclination of the canine femoral head and neck. Vet. Surg. 8, 74-77.

Jackson, G. M. and Wendelburg, K. L. (2012): Evaluation of the effect of distal femoral elevation on radiographic measurement of the anatomic lateral distal femoral angle. Vet. Surg. 41, 994-1001.

Lusetti, F., Bonardi, A., Eid, C., Brandstetter, A. and Martini, F. M. (2017): Pelvic limb alignment measured by computed tomography in purebred English Bulldogs with medial patellar luxation. Vet. Comp. Orthop. Traumatol. 30, 200-208.

Martins, J., Ferreira, A. J. and Ginja, M. M. (2012): Morphometric assessment of the hip joint in the Estrela Mountain Dog breed. Vet. Comp. Orthop. Traumatol. 25, 202-210.

Miles, J. E. (2016): Femoral rotation unpredictably affects radiographic anatomical lateral distal femoral angle measurements. Vet. Comp. Orthop. Traumatol. 29, 156-159.

Miles, J. E., Mortensen, M., Svalastoga, E. L. and Eriksen, T. (2015): A comparison of anatomical lateral distal femoral angles obtained with four femoral axis methods in canine femora. Vet. Comp. Orthop. Traumatol. 28, 193-198.

Montavon, P. M., Hohn, R. B., Olmstead, M. L. and Rudy, R. L. (1985): Inclination and anteversion angles of the femoral head and neck in the dog: evaluation of a standard method of measurement. Vet. Surg. 14, 277-282.

Nelson, L. L., Dyce, J. and Shott, S. (2007): Risk factors for ventral luxation in canine total hip replacement. Vet. Surg. 36, 644-653.

Noble, P. C., Alexander, J. W., Lindahl, L. J., Yew, D. T., Granberry, W. M. and Tullos, H. S. (1988): The anatomic basis of femoral component design. Clin. Orthop. Relat. Res. 235, $148-165$.

Ocal, M. K., Kara, M. E. and Turan, E. (2004): Computed tomographic measurements of the hip morphology of 10 healthy German Shepherd dogs. Vet. Rec. 155, 392-395.

Olmstead, M. L., Hohn, R. B. and Turner, T. M. (1983): A five-year study of 221 total hip replacements in the dog. J. Am. Vet. Med. Assoc. 183, 191-194.

Palierne, S., Asimus, E., Mathon, D., Meynaud-Collard, P. and Autefage, A. (2006): Geometric analysis of the proximal femur in a diverse sample of dogs. Res. Vet. Sci. 80, 243-252.

Palierne, S., Mathon, D., Asimus, E., Concordet, D., Meynaud-Collard, P. and Autefage, A. (2008): Segmentation of the canine population in different femoral morphological groups. Res. Vet. Sci. 85, 407-417.

Prieur, W. D. (1980): Coxarthrosis in the dog part I: Normal and abnormal biomechanics of the hip joint. Vet. Surg. 9, 145-149.

Sarierler, M. (2004): Comparison of femoral inclination angle measurements in dysplastic and nondysplastic dogs of different breeds. Acta Vet. Hung. 52, 245-252.

Savio, G., Baroni, T., Concheri, G., Baroni, E., Meneghello, R., Longo, F. and Isola, M. (2016): Computation of femoral canine morphometric parameters in three-dimensional geometrical models. Vet. Surg. 45, 987-995.

Schawalder, P. and Sterchi, H. P. (1981): Der Centrum-Collum-Diaphysenwinkel (CCD) und der Antetorsionswinkel (AT) beim Hund. II. Mitteilung: Korrelation zwischen dem CCD und dem AT. Röntgendiagnostische Aspekte. Kleintierpraxis 26, 273-278.

Sterchi, H. P. (1980): Winkelbestimmungen am proximalen Femurende. Beitrag zur Hüftgelenkbiomechanik des Hundes. PhD Thesis, Bern University.

Sumner, D. R., Devlin, T. C., Winkelman, D. and Turner, T. M. (1990): The geometry of the adult canine proximal femur. J. Orthop. Res. 8, 671-677.

Tomlinson, J., Fox, D., Cook, J. L. and Keller, G. G. (2007): Measurement of femoral angles in four dog breeds. Vet. Surg. 36, 593-598.

Tonnis, D. and Heinecke, A. (1999): Acetabular and femoral anteversion: relationship with osteoarthritis of the hip. J. Bone Joint Surg. Am. 81, 1747-1770. 